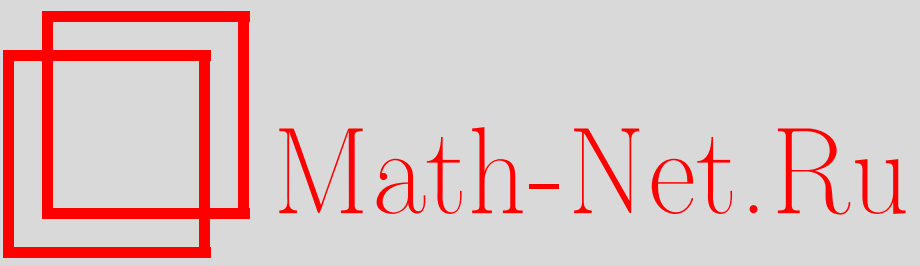

Н. Г. Мощевитин, О $\tau$-свойстве континуумов, $У М H, ~ 1999$, том 54, выпуск $3,169-$ 170

DOI: https://doi.org/10.4213/rm162

Использование Общероссийского математического портала Math-Net.Ru подразумевает, что вы прочитали и согласны с пользовательским соглашением http://www.mathnet.ru/rus/agreement

Параметры загрузки:

IP: 54.210 .77 .194

26 апреля 2023 г., 10:59:00 


\title{
О $\tau$-СВОЙСТВЕ КОНТИНУУМОВ
}

\author{
Н. Г. Мощевитин
}

1. Определение $\tau$-множества. Всюду ниже $M$-компактное, линейно связное метрическое пространство, состоящее более чем из одной точки, $d(\cdot, \cdot)$ - метрика.

ОПредЕЛЕнИЕ. Пусть $\tau>0$. Множество $A \subseteq M$ называется $\tau$-множеством, если оно может быть получено из некоторого линейно связного компакта $C \subseteq M$ посредством выкидывания счетного (или конечного, или пустого) семейства непересекающихся открытых множеств $\Delta_{j} \subset C$ так, что $A=C \backslash \bigcup_{j=0}^{\infty} \Delta_{j}, \Delta_{i} \cap \Delta_{j}=\varnothing$, причем для каждого "подходящего" множества $A_{\nu}=C \backslash \bigcup_{j=1}^{\nu} \Delta_{j}$ в его разложении на непустые непересекающиеся компоненты линейной связности $A_{\nu}=\bigcup_{\alpha} \Sigma_{\alpha, \nu}$ выполняется $\operatorname{diam} \Sigma_{\alpha, \nu}>\tau \operatorname{diam} \Delta_{\nu} \forall \nu \in \mathbb{N}, \forall \alpha$.

Диаметр пустого множества полагается равным нулю, так что любой неодноточечный линейно связный компакт в $M$ для каждого $\tau>0$ будет $\tau$-множеством.

ЗАмечАния. 1. $\tau$-множества на отрезке прямой рассматривались в работах [1]-[6].

2. Процесс получения $A$ из $C$ посредством выкидывания множеств $\Delta_{j}$, вообще говоря, нельзя вполне упорядочить по убыванию диаметров $\Delta_{j}$. В случае, когда $M$ есть отрезок вещественной прямой, это сделать можно (см. лемму из работы [5]), значит канторовы множества из [1]-[6] удовлетворяют нашему общему определению.

\section{2. Основная теорема.}

Tеорема. Пусть $A^{i}=C^{i} \backslash \bigcup_{j=1}^{\infty} \Delta_{j}^{i}, i=1,2$, суть $\tau_{1}-u \tau_{2}$-множества соответственно и выполнены условия

(i) $\tau_{1} \cdot \tau_{2} \geqslant 1$

(ii) $C^{i}$ не лежит целиком в одной компоненте линейной связности дополнения $M \backslash A^{k}, i \neq k$.

Тогда $A^{1} \cap A^{2} \neq \varnothing$.

ДокАЗАтельСтво. Рассмотрим компакт $C=C^{1} \cap C^{2}$. Предположим противное, полагая, что $C$ допускает покрытие $C \subset\left(\bigcup_{j=1}^{\infty} \Delta_{j}^{1}\right) \cup\left(\bigcup_{i=1}^{\infty} \Delta_{i}^{2}\right)$. Из этого покрытия выделяем конечное:

$$
C \subset\left(\Delta_{j_{1}}^{1} \cup \cdots \cup \Delta_{j_{p}}^{1}\right) \cup\left(\Delta_{i_{1}}^{2} \cup \cdots \cup \Delta_{i_{q}}^{2}\right) .
$$

Из условия (ii) следует, что $\min \{p, q\} \geqslant 1$. Будем считать, что из покрытия (1) нельзя выкинуть никакого $\Delta_{j}^{i}$ так, чтобы оставшиеся открытые множества составляли покрытие. Пусть

$$
\mu=\min \left\{\tau_{2}^{-1 / 2} \min _{1 \leqslant t \leqslant p} \operatorname{diam} \Delta_{j_{t}}^{1} ; \tau_{1}^{-1 / 2} \min _{1 \leqslant t \leqslant q} \operatorname{diam} \Delta_{i_{t}}^{2}\right\}
$$

Пусть для определенности $\mu=\tau_{2}^{-1 / 2} \operatorname{diam} \Delta_{j}^{1}$. Рассмотрим разбиение на компоненты линейной связности $C^{2} \backslash \bigcup_{t=1}^{q} \Delta_{i t}^{2}=\bigcup_{\alpha} \Sigma_{\alpha}$. Легко видеть, что

$$
\operatorname{diam} \Sigma_{\alpha}>\tau_{2} \min _{1 \leqslant t \leqslant q} \operatorname{diam} \Delta_{i_{t}}^{2}
$$

(когда $\operatorname{diam} \Delta_{j-1}<\operatorname{diam} \Delta_{j}$, то в процессе выкидывания их можно поменять местами, не нарушая $\tau$-свойства). Итак $\varnothing \neq C^{1} \cap\left(\bigcup_{\alpha} \Sigma_{\alpha}\right) \subset \bigcup_{t=1}^{p} \Delta_{j_{t}}^{1}$. Имеем два случая:

1) $\exists \alpha: \Sigma_{\alpha} \cap C^{1} \neq \varnothing, \Sigma_{\alpha} \nsubseteq C^{1}$, 2) $C^{1} \cap \Sigma_{\alpha} \neq \varnothing \Longrightarrow \Sigma_{\alpha} \subseteq C^{1}$.

В случае 1) $\partial C^{1} \cap \Sigma_{\alpha} \neq \varnothing$, но никакая точка границы $\partial \bar{C}^{1}$ не может принадлежать никакому $\Delta_{j}^{1}$ и имеем противоречие. В случае 2 ) из минимальности покрытия (1) и линейной связности каждого $\Sigma_{\alpha}$ выводим: $\forall t=1, \ldots, p \exists \alpha: \Sigma_{\alpha} \subset \Delta_{j_{t}}^{1}$. Теперь с учетом (3) получаем $\mu>\tau_{2}^{+1 / 2} \min _{1 \leqslant t \leqslant q} \operatorname{diam} \Delta_{i_{t}}^{2}$. Используя определение величины $\mu(2)$, выводим, что $\tau_{1} \cdot \tau_{2}<1$, что есть противоречие. Теорема доказана.

Работа выполнена при финансовой поддержке Российского фонда фундаментальных исследований (гранты №№ 96-01-00378, 96-15-96072). 
3. $\tau$-множества в экспоненциальном пространстве. Будем рассматривать линейно связное компактное пространство $\exp M=\{C \subseteq M: C$-компакт $\}$ с метрикой Хаусдорфа [7]. Обозначим

$$
\mathfrak{T}_{\tau}=\{A \subseteq M: A \text { является } \tau \text {-множеством }\}, \quad \mathfrak{T}=\bigcup_{\tau>0} \mathfrak{T}_{\tau} .
$$

УТВЕРЖДЕНИЕ 1. Т всюду плотно в $\exp M$.

Утверждение 1 очевидно и легко проверяется.

Точку $x \in M$ будем называть выводимой, если найдется ее открытая окрестность $B \ni x$ такая, что для любого $y \in B$ существуют $z \in M \backslash B$ и непрерьвная кривая $\gamma:[0 ; 1] \rightarrow M$ такие, что $\gamma(0)=x, \gamma(1 / 2)=y, \gamma(1)=z$ и функция $t \mapsto d(\gamma(t), x)$ не убывает на отрезке [0; 1$]$. Множество всех выводимых точек из $M$ будем обозначать $\mathscr{E}(M)$. Например, для куба $I=[0 ; 1]^{d}$ с евклидовой метрикой выполнено $\mathscr{E}(I)=I$. Отметим, что если $\mathscr{E}(M)$ всюду плотно в $M$, то T не имеет внутренних точек.

УТВеРЖДЕнИЕ 2. Пусть $\mathscr{E}(M)$ всюду плотно в $M$. Тогда для любого $\tau>0 \mathfrak{T}_{\tau}$ нигде не плотно в $\exp M$, и следовательно, $\mathfrak{T}$ в пространстве $\exp M$ имеет первую категорию Бәра.

Для доказательства утверждения 2 надо заметить, что в окрестности любого элемента $C \in$ $\exp M$ найдется элемент $C^{*}=\bigcup_{j=1}^{t} C_{j}$, представляющий собой объединение конечного числа компактов вида $C_{j}=\left(\overline{U_{\varepsilon}\left(x_{j}\right)} \backslash U_{\varepsilon / 2}\left(x_{j}\right)\right) \cup\left\{x_{j}\right\}$, где $x_{j}$ - выводимые точки, а через $U_{\lambda}\left(x_{j}\right)$ обозначена $\lambda$-окрестность точки $x_{j}$ в $M$ ( $\lambda$ мало). Ясно, что в малой окрестности множества $C^{*}$ в экспоненциальном пространстве не может находиться никакое $\tau$-множество с фиксированным $\tau$.

В экспоненциальном пространстве $\exp M$ также можно рассматривать $\tau$-множества. Будем называть $\tau$-множество $\mathfrak{W} \subset \exp M$ вырожденным, если дополнение $\mathfrak{D}=\exp M \backslash \mathfrak{W}$ линейно связно. Нетрудно видеть, что $\mathfrak{T}$ может содержать в себе некоторые подмножества $\mathfrak{W} \subset \mathfrak{T}$, которые являются вырожденными $\tau$-множествами.

УТвеРЖДЕнИЕ 3. Пусть $\mathscr{E}(M)=M$. Тогда $\mathfrak{T}$ не содержит в себе ни одного подмножества $\mathfrak{A} \subseteq \mathfrak{T}$, которое являлось бы невырожденным $\tau$-множеством.

ДоказАтельство. От противного. Невырожденность $\mathfrak{A}$ означает, что ехр $M \backslash \mathfrak{A}$ содержит два непересекающихся открытых множества $\mathfrak{D}_{0}, \mathfrak{D}_{1} \subset \exp M \backslash \mathfrak{A}$, участвующих в образовании $\tau$-структуры. Возьмем $C_{j} \in \mathfrak{D}_{j}, j=0,1$, которые представляют собой объединение конечного числа точек. $C_{0}$ можно непрерывно продеформировать в $C_{1}$ так, чтобы в каждый момент деформации компакт $C_{\lambda}, \lambda \in[0 ; 1]$, состоял из конечного числа точек. Отметим, что в $M$ никакое $\tau$-множество не может состоять из конечного числа точек (ибо $\mathscr{E}(M)=M)$. С другой стороны, существует $\lambda^{*} \in[0 ; 1]$ такое, что $C_{\lambda^{*}} \in \mathfrak{A} \subseteq \mathfrak{T}$. Противоречие. Утверждение доказано.

Автор благодарит Д. И. Савельева и А.М. Райгородского за многократные обсуждения настоящей работы.

\section{СПИСОК ЛИТЕРАТУРЫ}

[1] Hall M. Jr. // Ann. of Math. (2). 1948. V. 48. P. 966-993. [2] Newhouse S. // Proc. Sympos. Pure Math. 1970. V. 14. P. 191-203. [3] Newhouse S. // Topology. 1974. V. 13. P. 9-18. [4] Newhouse S. // Inst. Hautes Etudes Sci. Publ. Math. 1983. V. 50. P. 433-459. [5] Williams R. F. // Contemp. Math. 1991. V. 117. P. 163-175. [6] Мощевитин Н. Г. // УМН. 1997. Т. 52. № 6. С. 145-146. [7] Хаусдорф Ф. Теория множеств. М.-Л.: ОНТИ, 1937. 